\title{
An efficient approach to extract parameters from star cluster CMDs: fitCMD
}

\section{Charles Bonatto}

Departamento de Astronomia, Universidade Federal do Rio Grande do Sul, Av. Bento Gonçalves 9500 Porto Alegre $91501-970$, RS, Brazil

Accepted 2018 November 28. Received 2018 November 28; in original form 2018 October 17

\begin{abstract}
This work presents an approach ( $f i t C M D$ ) designed to obtain a comprehensive set of astrophysical parameters from colour-magnitude diagrams (CMDs) of star clusters. Based on initial mass function (IMF) properties taken from isochrones, fitCMD searches for the values of total (or cluster) stellar mass, age, global metallicity, foreground reddening, distance modulus, and magnitude-dependent photometric completeness that produce the artificial CMD that best reproduces the observed one; photometric scatter is also taken into account in the artificial CMDs. Inclusion of photometric completeness proves to be an important feature of fitCMD, something that becomes apparent especially when luminosity functions are considered. These parameters are used to build a synthetic CMD that also includes photometric scatter. Residual minimization between the observed and synthetic CMDs leads to the best-fitting parameters. When tested against artificial star clusters, fitCMD shows to be efficient in terms of both computational time and ability to recover the input values.
\end{abstract}

Key words: open clusters and associations: general.

\section{INTRODUCTION}

A colour-magnitude diagram (CMD) can be considered as the observational counterpart - on a purely photometric parameter space - of a stellar population (of given age and metallicity) whose individual masses are distributed according to an initial mass function (IMF). Over time, the mass-dependent stellar evolution changes the colour and magnitude of each star, thus leading to variations in the evolutionary sequences morphology. This reasoning implies that the CMD morphology encapsulates fundamental properties related to the star cluster itself, such as the stellar mass, age, metallicity, distance and reddening, among others.

The derivation of fundamental parameters of star clusters - in different dynamical states and formed in different environments is important for a wide variety of studies, ranging from determination of the star-formation rate in the Galaxy (e.g. Lamers \& Gieles 2006; Bonatto \& Bica 2011) to investigations on the infant mortality (e.g. Lada \& Lada 2003; Goodwin \& Bastian 2006) and the dynamical state and cluster dissolution time scales (e.g. Goodwin 2009; Lamers, Baumgardt \& Gieles 2010), among others. Broadly speaking, photometry is the easiest - and cheapest - way to collect data on substantial fractions of a star cluster's population. Transported to CMDs, the collected data then can be used to try and characterize the stellar population by extracting (some of) its fundamental parameters.

\footnotetext{
*E-mail: charles@if.ufrgs.br
}

However, the combination of limited photometric depth with distances in excess of a few Kpc usually prevents the fainter stars from being detected by most of the available large-scale photometric surveys, thus producing partially-sampled CMDs that, in turn, may lead to a deficient derivation of cluster parameters. Besides, the presence of unknown fractions of binaries, differential reddening (especially for the very young clusters), and photometric scatter and completeness, also add complications to the task of using CMDs to derive cluster parameters. In particular, while age, distance and reddening can be relatively easy to estimate even in CMDs that are somewhat noisy and do not contain the fainter stars, metallicity, and especially mass, remains way more elusive to measure.

Considering the potentially large scope that well-determined cluster parameters may have in the current Galactic astrophysics, several approaches have already been developed in order to circumvent the difficulties associated with the process of extracting fundamental parameters from CMDs, e.g. Naylor \& Jeffries (2006), Da Rio, Gouliermis \& Gennaro (2010), Stead \& Hoare (2011), and Bonatto, Lima \& Bica (2012). A summary of these approaches can be found in Bonatto et al. (2012). These approaches have been designed to deal with particular types of clusters and/or parameters, and some are not practical in terms of the required computational time.

The approach presented in Bonatto et al. (2012) included most of the relevant parameters (mass, age, star-formation spread, distance modulus, foreground and differential reddening, and binaries) that are expected to make up the CMDs of young clusters. In short, the idea was to find the parameters that produce a CMD that best 
reproduces the observed one. This was done by minimizing the residuals between the observed and simulated CMDs by means of a global optimization method that varied the parameters along the direction of the minimization. Although the approach was able to recover the input parameters for simulated CMDs, it required extremely long computational times when applied to actual star clusters. To minimize stochasticity, the approach worked with the average CMD of several mock clusters, each sharing the same set of parameters. Thus, the number of stars turned out to be very large, which in turn affected critically the computational time.

Based on lessons learned especially in Bonatto et al. (2012), a more efficient and comprehensive approach - fitCMD - to extract parameters from CMDs is here presented. Instead of working with mock star clusters, it is based on a mass function (of a given age, metallicity, and mass) displaced some distance from the Sun, and affected by reddening and photometric scatter. An additional feature is that now photometric completeness is also taken into account. It is shown to be efficient both in terms of computational time and recovery of input values from simulated CMDs. Similarly to Bonatto et al. (2012), the rationale involves the minimization of residuals between the simulated and observed Hess diagrams (Hess 1924).

This paper is organized as follows: Section 2 describes the approach; Section 3 discusses the efficiency of fitCMD in retrieving parameters from simulated CMDs; Section 4 discusses the application of fitCMD to actual CMDs of open clusters, a dwarf galaxy, and a globular cluster (GC). Concluding remarks are given in Section 5.

\section{THE APPROACH: FITCMD}

The basic goal of fitCMD is to extract fundamental parameters of a star cluster by means of the photometric information contained in its observed CMD. The underlying principle is to find an artificial CMD that best reproduces the observed one; the set of input parameters is then assumed to be representative of the star cluster itself. The parameters included here are the total mass stored in stars (or cluster mass, $M_{\mathrm{cl}}$ ), age $\left(t_{\mathrm{age}}\right)$, global metallicity $(Z)$, foreground reddening (or colour excess, $C E$ ) and the apparent distance modulus $(D M)$. Photometric completeness is also taken into account by means of a Fermi function. Completeness as a function of the magnitude $m$ is described as $f_{\mathrm{C}}(m)=1 /\left(1+\exp \left[k_{F}\left(m-m_{\mathrm{TO}}\right)\right]\right)$, where $m_{\mathrm{TO}}$ is the turnover magnitude $\left(f_{\mathrm{C}}\left(m_{\mathrm{TO}}\right)=0.5\right)$ and $k_{F}$ controls the steepness of the descent. Strictly speaking, this formulation refers to the completeness (at magnitude $m$ ) relative to that of the brightest stars. The analytical shape of the Fermi function appears to be convenient to describe the photometric completeness because it changes rather slowly at the bright end, and falls off exponentially after some fainter magnitude. When completeness effects are irrelevant, the stellar density over the evolutionary sequences of a stellar population on a CMD should follow approximately that of a mass function. However, completeness artificially decreases the stellar density towards fainter magnitudes, and the comparison with the intrinsic density allows fitCMD to derive the completeness function parameters $k_{F}$ and $m_{\mathrm{TO}}$.

If binaries and differential reddening are ignored, an artificial star cluster CMD can be built by defining the following minimum set of parameters: $M_{\mathrm{cl}}, t_{\mathrm{age}}, Z, C E$ and $D M$. Individual stellar masses are attributed according to an IMF, which can be chosen either as that of Salpeter (1955) or the segmented distribution of Kroupa (2001). Photometric scatter must also be added for more realism. In addition, a mass to light relation (MLR) - usually taken from isochrones - is required to produce the CMD representation of a mock star cluster. Examples of such experiments are given in e.g. Bonatto et al. (2012).

Regarding isochrones, this work employs the latest PARSEC ${ }^{1}$ v1.2S+COLIBRI PR16 (Bressan et al. 2012; Marigo et al. 2017) models, although any other system having an adequate coverage in age, metallicity and stellar mass can be used. The present work employs filters that are part of the following photometric systems (i) Bessell (1990) and Bessell \& Brett (1988) (filters B, $V, J$, and $K$ ), (ii) DECAM (filters $g$ and $r$ ), and (iii) WFC/ACS (filters $F 606 \mathrm{~W}$ and $F 814 \mathrm{~W}$ ). These isochrones contain stars with masses larger than $0.1 \mathrm{M}_{\odot}$ and are computed for a scaled-solar composition, and follow the relation $Y=0.2485+1.78 Z$, where $Y$ is the $\mathrm{He}$ content and $Z$ is the total metallicity; the solar metal content is $Z_{\odot}=0.0152$. They also include the pre-mainsequence phase. For an adequate coverage in age, the following values are considered: $1-10 \mathrm{Myr}$ (in steps of $\Delta t=1 \mathrm{Myr}$ ), $10-20 \operatorname{Myr}(\Delta t=2 \mathrm{Myr}), 20-50 \mathrm{Myr}(\Delta t=5 \mathrm{Myr}), 50-100 \mathrm{Myr}$ $(\Delta t=10 \mathrm{Myr}), 100-500 \mathrm{Myr}(\Delta t=25 \mathrm{Myr}), 500-1000 \mathrm{Myr}$ ( $\Delta t=50 \mathrm{Myr}), 1000-13500 \mathrm{Myr}(\Delta t=250 \mathrm{Myr})$. In terms of the metallicity $Z$, the sampling is: from $10^{-4}$ to $10^{-3}$ (steps of $\left.\Delta Z=10^{-4}\right)$, and $10^{-3}$ to $3 \times 10^{-2}\left(\Delta Z=10^{-3}\right)$. As a consequence of such a relatively high resolution in age and metallicity, the total number of isochrones is 3978 , but smaller ranges can be considered as well (see below).

Consider an artificial star cluster $\left(\mathrm{SC}_{O}\right)$ characterized by the set of intrinsic parameters $\left(M_{\mathrm{cl}}, t_{\mathrm{age}}, Z, I M F\right)$. Obviously, any other model star cluster built with the same parameters will be a twin representation of $\mathrm{SC}_{O}$. But, given the statistical nature of the individual stellar masses distribution, and, to some extent, their colours and magnitudes, the corresponding CMD may end up presenting significant differences with respect to that of $S C_{O}$, especially for young and low-mass cases (e.g. Bonatto et al. 2012). However, the fundamental point here is that the IMF is the same in all cases. So, instead of the discrete - and stochastic - CMDs, the present approach is based on properties of the IMF itself; a brief description is sketched below.

The first step consists in defining the ranges in which the parameters $\left(M_{\mathrm{cl}}, t_{\mathrm{age}}, Z, C E, D M\right)$ will be searched. This, in turn, also sets the actual number of isochrones to be dealt with. With the IMF, a CMD is built for each isochrone (at $D M=0$ and no reddening), with a discretization in the colour/magnitude plane also defined at the beginning. So, a given CMD cell - containing stars with mass in the range $(m 1, m 2)$ - will have the number of stars per cluster mass $n_{H}$ $=N_{1,2} / M_{\mathrm{cl}}$, as well as the average mass $(<m>)$, which basically involves integrating the mass function $[\phi(m)=d N / d m)]$ between $(m 1$, $m 2): N_{1,2}=\int_{m 1}^{m 2} \phi(m) \mathrm{d} m$. This operation is done a single times and before the parameter search, which drastically reduces the computational time. After these procedures, each CMD cell will contain the respective relative density (number per cluster mass) of occurrence of stars, which is equivalent to the classical Hess diagram; hereafter, these diagrams are denoted by $H_{M}=H_{M}\left(M_{\mathrm{cl}}, t_{\text {age }}, Z\right)$. This process is illustrated in Fig. 1. The same discretization is then applied to the observed star cluster CMD when building the respective Hess diagram. Photometric uncertainties are explicitly taken into account in the observed Hess diagram (e.g. Bonatto et al. 2012).

Photometric scatter is another key component of any artificial CMD that is expected to reproduce that of a star cluster. Consider the rows corresponding to the magnitude in the observed Hess diagram. For each row we compute the total stellar density and build the cor-

\footnotetext{
${ }^{1}$ Downloadable from http://stev.oapd.inaf.it/cgi-bin/cmd
} 

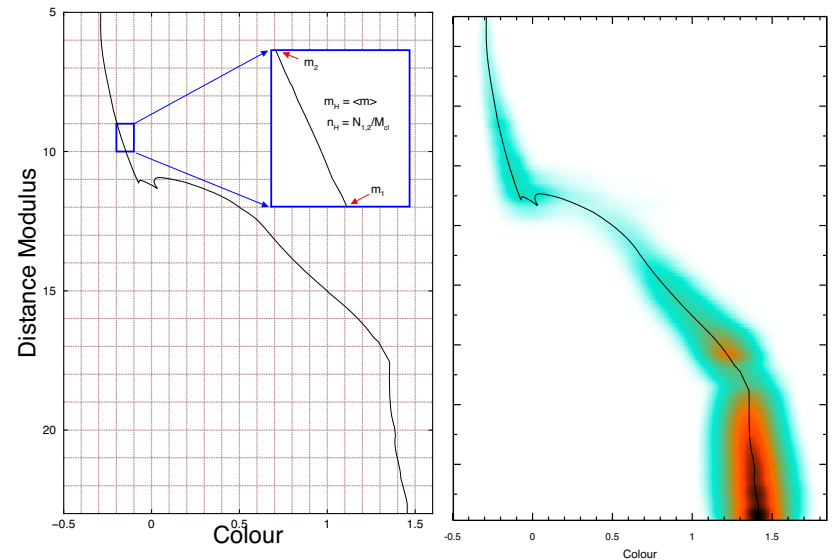

Figure 1. Left panel: given an IMF (represented by an isochrone), a CMD cell will contain $N_{1,2}$ stars with mass in the range $(m 1, m 2)$, with an average stellar mass $<m>$; the stellar density is defined as $n_{H}=N_{1,2} / M_{\mathrm{cl}}$. Right: Hess diagram - with photometric scatter added - of the CMD at left.

responding distribution function as a function of colour (columns). To minimize local fluctuations, the stellar density function for row $N$ is actually the average of those in rows $N-1, N$ and $N+1$. After identifying the colour where the maximum occurs, we compute the fraction of the total stellar density that each colour cell contains, both for bluer and redder colours with respect to the maximum. This broadening function is then applied to the respective row (magnitude value) in the simulated Hess diagram (see below). Thus, fitCMD allows both for colour-asymmetric and magnitudedependent scatter. Comparisons of the actual photometric scatter with the simulated ones can be seen in Figs 2-4.

The parameter search consists of finding the absolute minimum of the residual hyper-surface $\left(R_{H}\right)$ defined as $R_{H}=\left(H_{\mathrm{obs}}-H_{\text {sim }}\right)^{2}$, where $H_{\mathrm{obs}}$ is the observed Hess diagram and $H_{\text {sim }}=H_{\text {sim }}\left(M_{\mathrm{cl}}, t_{\mathrm{age}}\right.$, $\left.Z, C E, D M, k_{F}, m_{\mathrm{TO}}\right)$ is the simulated one. The values of $M_{\mathrm{cl}}, t_{\mathrm{age}}$, $Z, C E, D M, k_{F}$, and $m_{\mathrm{TO}}$ at the absolute minimum are assumed to represent those of the star cluster. In practical terms, locating the minima of $R_{H}$ is equivalent to finding the parameters that minimize the quantity

$S_{R}=\sum_{\mathrm{mag}, \mathrm{col}} W(\mathrm{mag}) \times R_{H}(\mathrm{mag}, \mathrm{col})$,

on the colour/magnitude plane. The sum runs over all Hess cells and $W(\mathrm{mag})$ is the statistical weight of each cell. $W(\mathrm{mag})$ corresponds to the inverse of the observed Hess density computed at the respective magnitude of each cell, i.e. $W(\mathrm{mag})=1 / \sum_{\mathrm{col}} H_{\mathrm{obs}}(\mathrm{mag}, \mathrm{col})$.

We employ the global optimization method known as Simulated Annealing (SA; Goffe, Ferrier \& Rogers 1994). Simulated annealing originates from the metallurgical process by which the controlled heating and cooling of a material is used to increase the size of its crystals and reduce their defects. If an atom is stuck to a local minimum of the internal energy, heating forces it to randomly wander through higher energy states. In the present context, a state is the surface $R_{H}=R_{H}\left(M_{\mathrm{cl}}, t_{\text {age }}, Z, C E, D M, k_{F}, m_{\mathrm{TO}}\right)$, corresponding to a specific set of values of the parameters being optimized. The slow cooling increases the probability of finding states of lower energy than the initial one. SA is a global optimization technique that can escape from local minima (Goffe et al. 1994; Bonatto et al. 2012).

$\mathrm{SA}$ is an iterative and statistical technique that, at any given step $k$, randomly selects a new set of parameters $\left(M_{\mathrm{cl}}^{k}, t_{\mathrm{age}}^{k}, Z^{k}, C E^{k}\right.$, $D M^{k}, k_{F}^{k}, m_{\mathrm{TO}}^{k}$ ) from the respective ranges. This also means that the
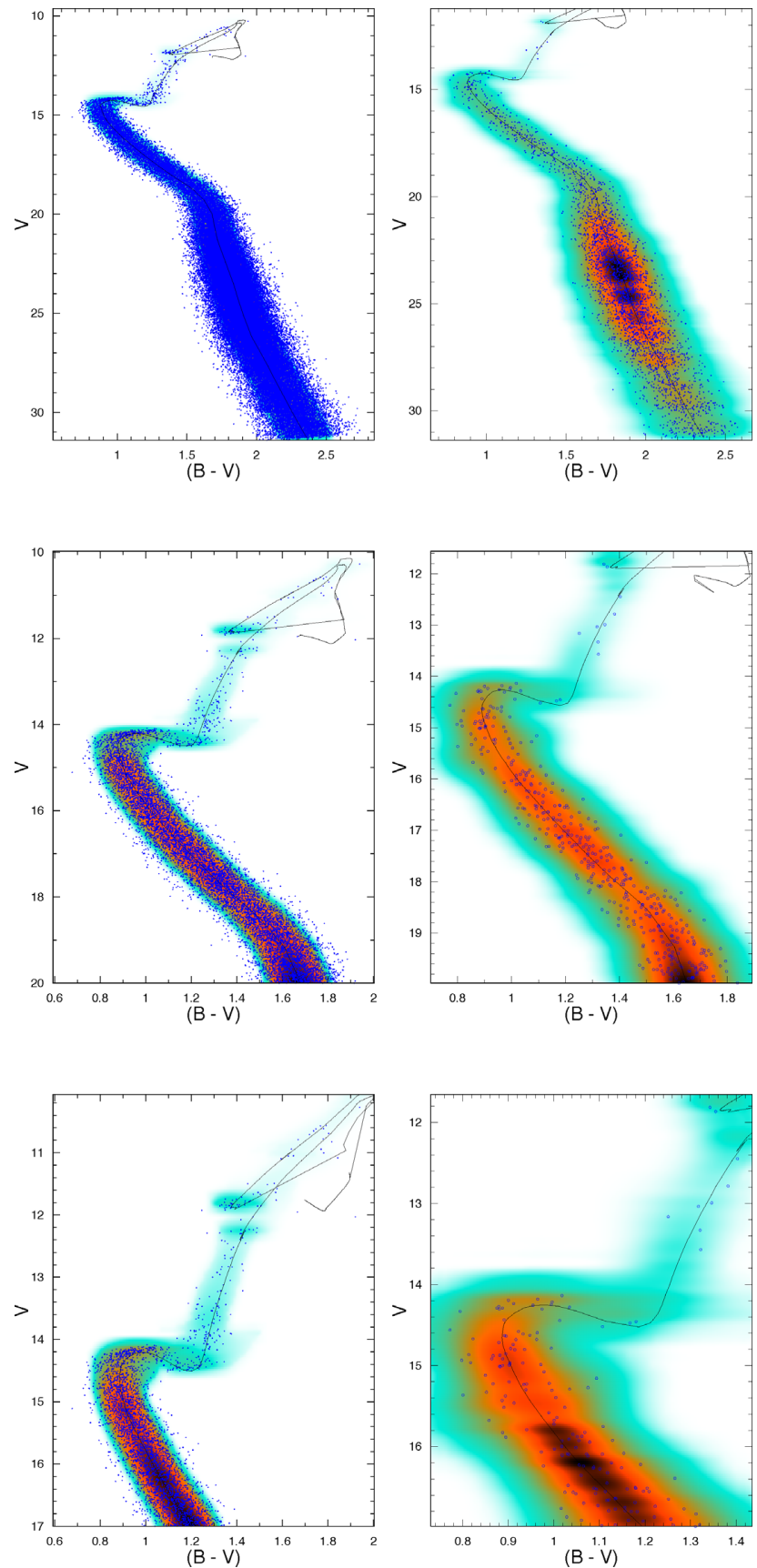

Figure 2. CMDs (blue dots) of simulated star clusters with $M_{\mathrm{cl}}=$ $2 \times 10^{4} \mathrm{M}_{\odot}$ (left panels) and $M_{\mathrm{cl}}=1 \times 10^{3} \mathrm{M}_{\odot}$ (right). They are analysed by fitCMD over the full magnitude range (top panels), and partial ranges (middle and bottom). The best solutions are shown as Hess diagrams, in which the corresponding isochrone is set.

corresponding diagram $H_{M}^{k}=H_{M}\left(M_{\mathrm{cl}}^{k}, t_{\mathrm{age}}^{k}, Z^{k}\right)$ will be the only one to be used in this particular iteration. The cells of $H_{M}^{k}$ are then multiplied by $M_{\mathrm{cl}}^{k}$ (to end up containing the number density of stars - same as $H_{\mathrm{obs}}$ ), and shifted by the values of $D M^{k}$ and $C E^{k}$. Finally, the artificial stellar density of each cell is decreased according to the photometric completeness of the corresponding magnitude, and photometric scatter is added (see above). What results is the diagram $H_{\text {sim }}$, from which $R_{H}$ is built. As $S_{R}$ decreases, i.e. a new minimum is found, SA concentrates on smaller parameter ranges, centred around the most promising values, and a new step $(k+1)$ is taken. Iterations 

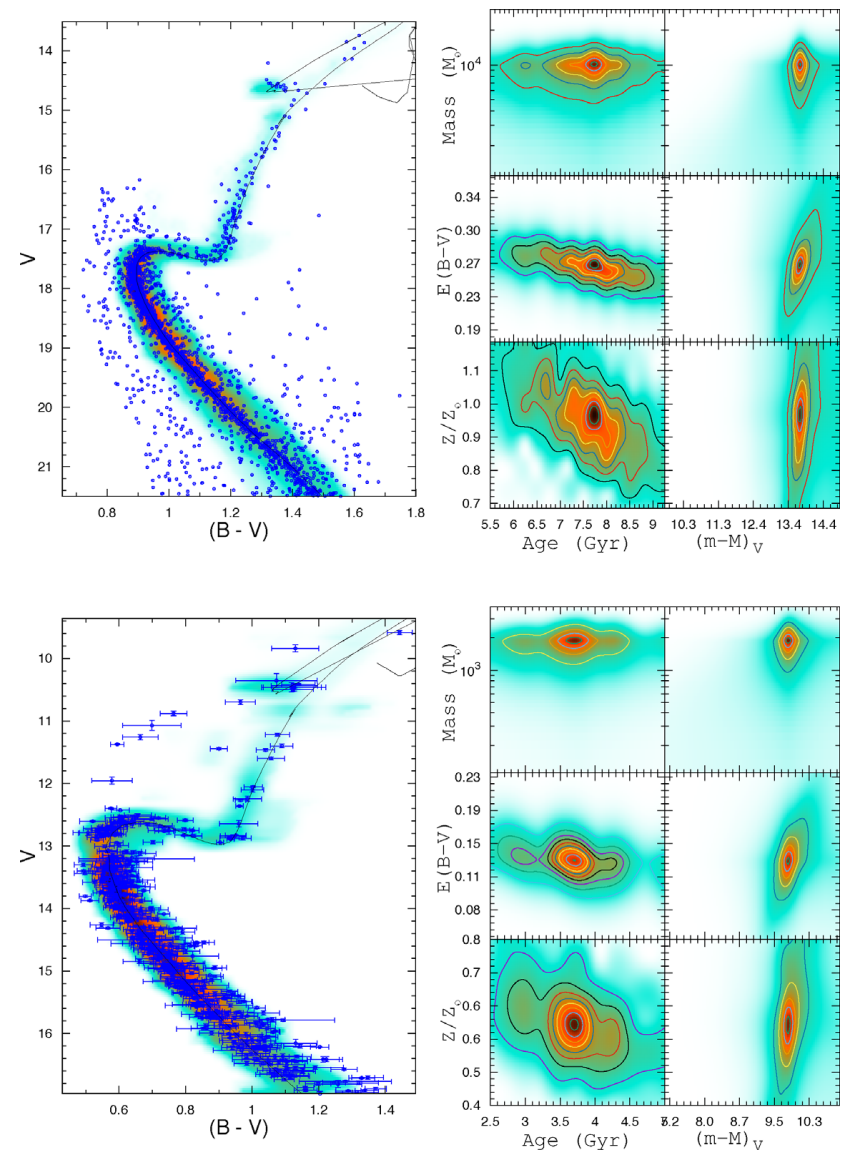

Figure 3. Left panels: same as Fig. 2 for the old OCs NGC 6791 (top) and NGC 2682 (bottom). The respective solution maps are shown in the right panels.

stop when SA meets the convergence criterion: five consecutive repetitions of the same value of $S_{R}$. Typical runtimes are around 1 min on an Intel Core $7920 @ 2.67$ GHz processor. However, given the statistical nature of SA, fitCMD should be repeated a few times to minimize the probability of getting stuck into a deep, but secondary minimum.

An interesting aspect of fitCMD is that, if the mass distribution of a cluster follows relatively closely a MF, the cluster mass should be retrievable quite well even when working with CMDs not severely truncated by limited photometric depth. In other words, if the age, metallicity, distance modulus and colour excess are reasonably well determined, the ratio observed/artificial Hess cells content - in any magnitude range - corresponds to $M_{\mathrm{cl}}$.

\section{TESTING fitCMD ON ARTIFICIAL CMDS}

The efficiency of fitCMD in recovering input parameters is tested with simulated CMDs, built with pre-defined values of $M_{\mathrm{cl}}, t_{\text {age }}$, $Z, C E$, and $D M$. Individual stellar masses are attributed according to Kroupa's IMF (Kroupa 2001) - for masses larger than $0.1 \mathrm{M}_{\odot}-$ until the sum matches $M_{\mathrm{cl}}$; the respective magnitudes are taken from the PARSEC isochrone corresponding to $t_{\text {age }}$ and $Z$. Typical photometric uncertainties (for the simulated distance from the Sun) are added for each band $k\left(\sigma_{k}\right)$. For more realism, the final photometric values for each star in band $k$ correspond to those in the isochrone $\left(m_{k}\right)$ plus a displacement that is taken from a Gaussian distribution
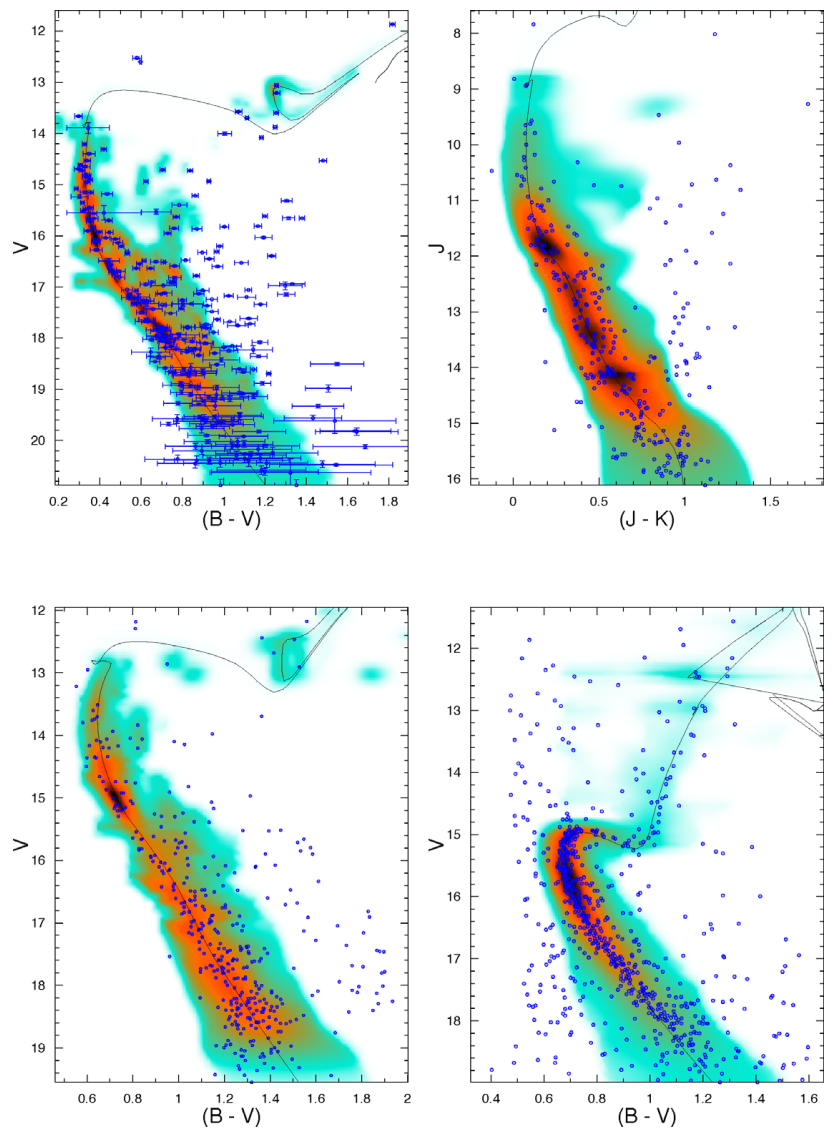

Figure 4. Same as Fig. 2 for NGC 2635 (top-left), NGC 2323 (top-right), NGC 5288 (bottom-left), and NGC 188 (bottom-right).

centred on $m_{k}$ and having the standard deviation $\sigma_{k}$, thus emulating observational photometric scatter.

CMDs dealt with here correspond to the classical $V \times(B-V)$. For simplicity, we consider two models with identical age $\left(t_{\text {age }}=\right.$ $5 \mathrm{Gyr})$, apparent distance modulus $\left[(m-M)_{V}=10.94\right]$, colour excess $[E(B-V)=0.3]-$ thus corresponding to $\mathrm{d}_{\odot}=1 \mathrm{kpc}$, and global metallicity $\left(Z / Z_{\odot}=1.18\right)$, but with very different masses $\left(M_{\mathrm{cl}}=2 \times 10^{4} \mathrm{M}_{\odot}\right.$ and $\left.M_{\mathrm{cl}}=1 \times 10^{3} \mathrm{M}_{\odot}\right)$ and, consequently, number of stars present in the CMD. Effects of photometric depth on the recovered parameters are also considered (Table 1). At $\mathrm{d}_{\odot}$ $=1 \mathrm{kpc}$, the mass distribution would reach a magnitude limit of $V \lesssim 31$. Then, to emulate photometric depth, we also consider CMDs restricted to $V \leq 20$ and $V \leq 17$.

Results obtained by fitCMD can be seen in Fig. 2 and are quantified in Table 1. The first point is that fitCMD does recover the input values - within uncertainties - even for the severely depleted CMDs. As expected, the cluster mass determination does not depend on photometric depth and/or number of stars present in the CMD. Age and metallicity, on the other hand, are more sensitive, especially to the evolutionary sequence tightness, i.e. essentially the number of stars.

\section{APPLICATION TO ACTUAL STELLAR SYSTEMS}

After exploring the ability of fitCMD to recover input model parameters, we now apply it to CMDs of actual star clusters. For this we selected some OCs with photometry publicly available in 
Table 1. Recovery of model parameters.

\begin{tabular}{|c|c|c|c|c|c|c|c|}
\hline $\begin{array}{l}\text { Range } \\
\text { (mag) } \\
(1)\end{array}$ & $\begin{array}{c}N \\
\text { (stars) } \\
(2)\end{array}$ & $\begin{array}{c}M_{\mathrm{cl}} \\
\left(\mathrm{M}_{\odot}\right) \\
(3)\end{array}$ & $\begin{array}{c}t_{\mathrm{age}} \\
(\mathrm{Gyr}) \\
(4)\end{array}$ & $\begin{array}{c}(m-M)_{V} \\
(\mathrm{mag}) \\
(5)\end{array}$ & $\begin{array}{c}E(B-V) \\
(\mathrm{mag}) \\
(6)\end{array}$ & $\begin{array}{c}Z / Z_{\odot} \\
(7)\end{array}$ & $\begin{array}{c}M_{\mathrm{CMD}} \\
\left(\mathrm{M}_{\odot}\right) \\
(8)\end{array}$ \\
\hline Full & 61034 & $20000_{-598}^{+642}$ & $5.0_{-0.4}^{+0.3}$ & $10.94_{-0.03}^{+0.03}$ & $0.30_{-0.01}^{+0.01}$ & $1.18_{-0.04}^{+0.02}$ & 20000 \\
\hline$V<20$ & 8643 & $20000_{-1590}^{+1870}$ & $5.0_{-0.17}^{+0.24}$ & $10.94_{-0.02}^{+0.03}$ & $0.30_{-0.0}^{+0.01}$ & $1.18_{-0.03}^{+0.04}$ & 7400 \\
\hline Model & 3103 & 1000 & 5 & 10.94 & 0.30 & 1.18 & 1000 \\
\hline Full & 3103 & $993_{-84}^{+98}$ & $4.8_{-1.1}^{+1.0}$ & $10.89_{-0.07}^{+0.09}$ & $0.30_{-0.01}^{+0.01}$ & $1.25_{-0.07}^{+0.07}$ & 990 \\
\hline$V<20$ & 416 & $991_{-145}^{+184}$ & $4.8_{-0.8}^{+0.4}$ & $10.93_{-0.06}^{+0.05}$ & $0.30_{-0.01}^{+0.01}$ & $1.18_{-0.06}^{+0.01}$ & 360 \\
\hline$V<17$ & 184 & $1010_{-132}^{+244}$ & $5.0_{-0.2}^{+0.0}$ & $10.94_{-0.06}^{+0.06}$ & $0.31_{-0.01}^{+0.02}$ & $1.05_{-0.02}^{+0.04}$ & 190 \\
\hline
\end{tabular}

Notes. Col. (1) - magnitude threshold considered; (2) - number of stars present in the CMD; (3) - derived cluster mass; (4) - age; (5) - distance modulus; (6) - colour excess; (7) - metallicity; (8) - stellar mass actually present in CMD.

VizieR, ${ }^{2}$ NGC 6791, NGC 188, NGC 2682, NGC 2635, NGC 5288, and NGC 2323. Some details and values of parameters recently derived for these OCs are provided below.

NGC 6791: BVR photometry from Montgomery, Janes \& Phelps (1994). A recent review on properties on this old and relatively metal-rich OC is in Martinez-Medina et al. (2018). Some relevant parameters are an age in the range $6-8 \mathrm{Gyr},[\mathrm{Fe} / \mathrm{H}] \approx+0.4$, $\mathrm{d}_{\odot} \approx 4 \mathrm{kpc}$, and $M_{\mathrm{cl}} \sim 5000 \mathrm{M}_{\odot}$.

NGC 188: BVI photometry from Sarajedini et al. (1999). The review by Hills et al. (2015) provides the following parameter ranges, $t_{\text {age }}=5.8$ to $6.5 \mathrm{Gyr},[\mathrm{Fe} / \mathrm{H}]=-0.77$ to $+0.125,(m-M)_{V}=$ 11.441 to 11.525 , and $A_{V}=0.162$ to 0.236 .

NGC 2682 (M 67): BVR photometry from Yadav et al. (2008). Available parameters in the literature: $t_{\mathrm{age}} \sim 4 \mathrm{Gyr}, M_{\mathrm{cl}} \sim 2000 \mathrm{~ms}$ (Hurley et al. 2005), $[\mathrm{Fe} / \mathrm{H}]=0.00 \pm 0.06$ (Heiter et al. 2014), $E(B$ $-V)=0.041 \pm 0.004$ (Taylor 2007).

NGC 2635: BVI photometry from Moitinho et al. (2006), which also provides the values $t_{\text {age }} \sim 600 \mathrm{Myr}, E(B-V)=0.35$, $\mathrm{d}_{\odot} \sim 4 \mathrm{kpc}$, and $Z \sim 0.004$.

NGC 5288: BVI photometry from Piatti, Clariá \& Ahumada (2006), which also provides the values $t_{\mathrm{age}} \sim 130 \mathrm{Myr}, E(B-V)=$ $0.75, \mathrm{~d}_{\odot}=2.1 \pm 0.3 \mathrm{kpc}$, and $Z \sim 0.04$.

NGC 2323: $\mathrm{JHK}_{S}$ photometry from 2MASS Skrutskie et al. (2006); parameters in the literature are $t_{\text {age }}=140 \pm 20 \mathrm{Myr}, \mathrm{d}_{\odot}=$ $115 \pm 20 \mathrm{pc},[\mathrm{Fe} / \mathrm{H}]=0.00$, and $E(B-V)=0.23 \pm 0.06$ (Cummings et al. 2016); $t_{\text {age }}=140 \pm 20 \mathrm{Myr}, \mathrm{d}_{\odot}=900 \pm 100 \mathrm{pc}$, and $M_{\mathrm{cl}} \sim 890 \mathrm{M}_{\odot}$ (Amin \& Elsanhoury 2017).

As a caveat, it is important to remark that, except for NGC 2323, the remaining OCs may contain varying fractions of field stars contaminating their CMDs, which may lead to extrinsic stellar-density differences between the observed and IMF-simulated evolutionary sequences. The wide-field 2MASS photometry of NGC 2323 (obtained from VizieR) has been field-star cleaned with the decontamination algorithm described in Bonatto \& Bica (2007), which employs a comparison field containing a statistically significant number of stars. Photometry for the other OCs, on the other hand, was obtained from specific observational projects, and usually corresponds to stars located within the cluster radius, thus with no comparison field available. Nevertheless, they provide interesting

${ }^{2}$ http://vizier.u-strasbg.fr/viz-bin/VizieR cases in which fitCMD can be tested under realistic conditions and those usually found in star clusters.

In all cases the age and metallicity parameters were allowed to vary over their whole ranges (Section 2); the remaining search parameters were also let to vary over broad ranges. For comparison reasons, simulations not taking photometric completeness into account were also performed and will be discussed later. The observed CMDs together with the respective fitCMD solutions are shown in Figs 3 and 4; to minimize clutter, error bars are not shown in the cases where the OC has many stars. The best-fitting and additional derived parameters are given in Table 2, among them is the stellar mass actually present in the CMD $\left(M_{\mathrm{CMD}}\right)$, which in some cases corresponds to a fraction of the total mass $\left(M_{\mathrm{cl}}\right)$.

After finding the parameters corresponding to the absolute minimum of the $R_{H}$ surface, their uncertainties are computed by means of the shape of the solution well in selected 2D projections (or solution maps). For instance, the solution map for mass and age is built by setting $D M, Z, C E, k_{F}$, and $m_{\mathrm{TO}}$ to their optimum values, while keeping $M_{\mathrm{cl}}$ and $t_{\text {age }}$ free to vary within the pre-defined ranges. Uncertainties - usually asymmetric - are then computed based on properties (depth and width of the absolute minimum) of the solution map. Examples of solution maps are given in Fig. 3 for NGC 6791 and NGC 2682.

Besides the magnitude values as a function of mass, the PARSEC isochrones provide additional data that allow us to compute interesting star cluster integrated quantities, such as the bolometric and the absolute magnitude in the $V$ band, and the mass-to-light ratio (MLR) both bolometric and in $V$ (Table 2).

A few comments on the results obtained with fitCMD for the selected OCs. A direct comparison of all the output parameters from fitCMD with those in the literature cannot be done, since usually each work is aimed at a particular parameter (or restricted set of parameters). Nevertheless, the fitCMD ages agree - within the uncertainties - with those in the literature for NGC 6791, NGC 188 and NGC 2682, but differ somewhat for the remaining OCs. Concerning $M_{\mathrm{cl}}$, our values for NGC 2682 and NGC 2323 agree with previous ones, but we find a value twice as large for NGC 6791. In summary, fitCMD provides values for a set of interesting astrophysical parameters obtained in a self-consistent way.

The two free parameters related to the photometric completeness are given in Table 2, and the completeness functions derived for the sample OCs are shown in Fig. 5. Among the selected OCs, 

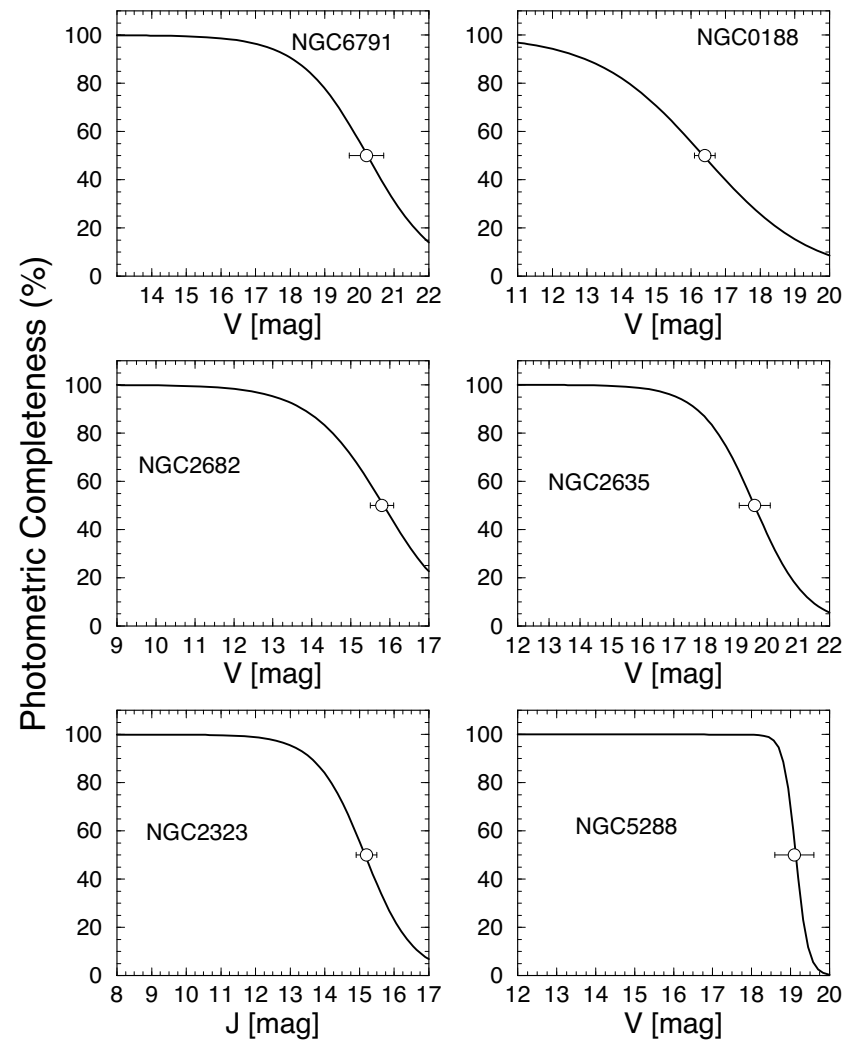

Figure 5. Photometric completeness $\left(f_{\mathrm{C}}\right)$ as a function of magnitude $(m)$ : $f_{\mathrm{C}}(m)=1 /\left(1+\exp \left[k_{F}\left(m-m_{\mathrm{TO}}\right)\right]\right)$. Turnover magnitude $\left(m_{\mathrm{TO}}\right)$ is shown by the open circle.

NGC 188 is the one that suffers the highest degree of photometric completeness, beginning even at the brightest observed magnitude ( $V \sim 11$ and $m_{\mathrm{TO}} \approx 16.4$ ). NGC 5288, on the other hand, is the least affected, with completeness only affecting stars fainter than $V \sim 18$, with $m_{\mathrm{TO}} \approx 19.1$.

Another way to examine the results is by comparing the observed luminosity function (LF) with that measured on the CMD built with the best-fitting parameters (Fig. 6). The LFs are built by simply integrating the stellar density across the magnitude axis, thus implying that some contribution from contaminant stars may be present in the observed LFs. For a cleaner visualization, Fig. 6 shows the $1 \sigma$ bounds of the observed LFs. In general, fitCMD reproduces the observed LFs, within the uncertainties, even at the faint end where completeness is more important. For comparison purposes, Fig. 6 also shows the LF produced by fitCMD when photometric completeness is not taken into account (red line). Significant deviations occur with respect to both the complete and observed LFs, especially at the faint magnitude ranges for the OCs most affected by completeness. This occurs because fitCMD tries to match the observed stellar density in all cells according to an IMF. Since photometric completeness naturally decreases the stellar densities (especially at the faint end of CMDs), fitCMD also lowers the stellar density in brighter cells. Finally, Fig. 6 shows the completeness-corrected LFs (blue line).

\subsection{Special cases}

After being applied to artificial and classical OCs, it would be interesting to check the performance of fitCMD on potentially more difficult cases. To this end, the Milky Way satellite Reticulum II and 

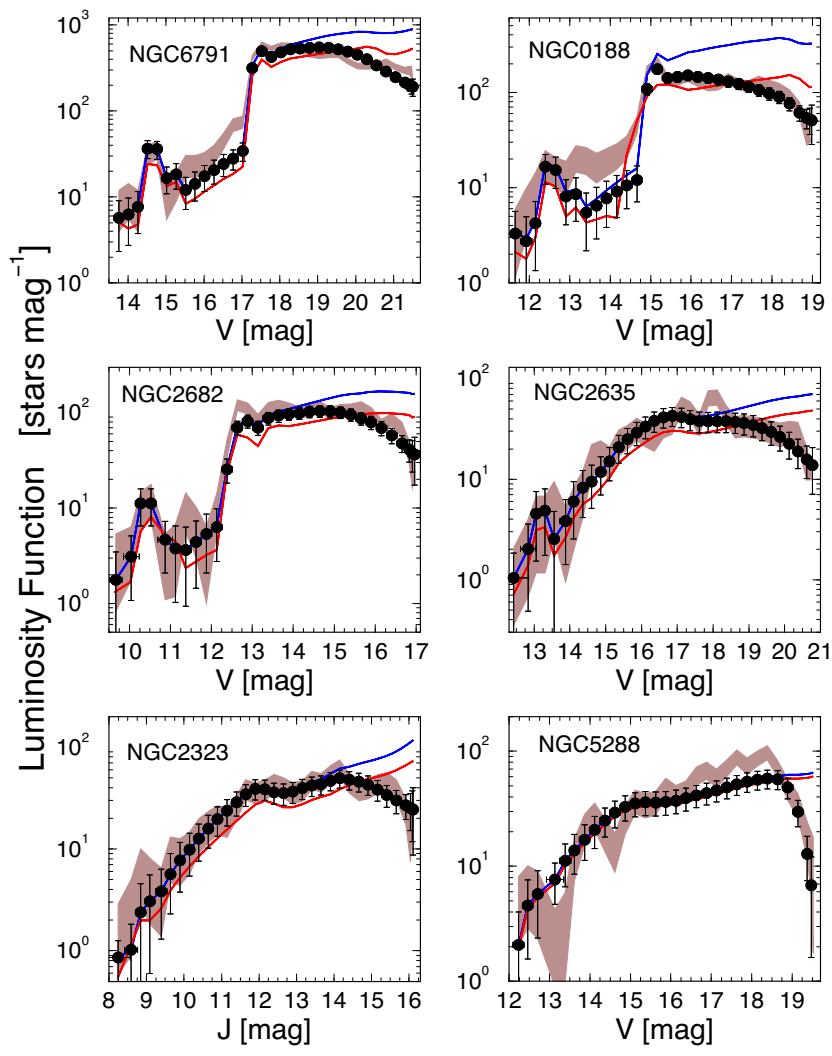

Figure 6. Luminosity functions. Shaded region: $1 \sigma$ bounds of the observed LF; black symbols: fitCMD simulated LF; blue solid line: completenesscorrected LF; red line: best-fitting LF without considering completeness.

the GC 47 Tuc have been selected. Both objects contain mixtures of stellar populations (see below) probably characterized by different ages and metallicities.

The dwarf galaxy Ret II (DES J0335.6-5403) was discovered by the Dark Energy Survey (DES; Bechtol et al. 2015). Parameters for Ret II derived by Bechtol et al. (2015) are the half-light radius $R_{\mathrm{hl}} \approx 6$ arcmin, distance to the Sun $\mathrm{d}_{\odot}=32 \mathrm{kpc}$, stellar mass $M_{\mathrm{cl}}=(2.6 \pm 0.2) \times 10^{3} \mathrm{M}_{\odot}$, absolute magnitude $M_{V}=$ $-3.6 \pm 0.1$, age $=10 \pm 5 \mathrm{Gyr}$, and the global metallicity $Z<0.0003$. More recently, Mutlu-Pakdil et al. (2018) studied Ret II with deep Magellan/Megacam photometry (also for stars within $R_{\mathrm{hl}}$ ), finding $\mathrm{d}_{\odot}=31.4 \pm 1.4 \mathrm{kpc}, M_{V}=-3.1 \pm 0.1$, age $=13.5 \mathrm{Gyr}$, and $[\mathrm{Fe} / \mathrm{H}]$ $=-2.4$; for $0.0<[\alpha / \mathrm{Fe}]<0.4$, the total metallicity of Ret II would be $Z<0.0001$, consistent with the value derived by Bechtol et al. (2015).

Photometry for Ret II stars in $g$ and $r$ bands (corresponding to the DES system) has been obtained from the NOAO Data $\mathrm{Lab}^{3}$ in a region of 30 arcmin radius around its central coordinates, $\operatorname{RA}(J 2000)=03^{h} 35^{\prime} 49^{\prime \prime}$ and $\operatorname{DEC}(J 2000)=-54^{\circ} 02^{\prime} 48^{\prime \prime}$. This setup was necessary in order to produce a field-stars decontaminated CMD by means of the Bonatto \& Bica (2007) algorithm. For consistency and comparison purposes with both previous works, the region analysed here corresponds to the area within $R_{\mathrm{hl}}$. The decontaminated CMD together with the fitCMD solution are shown in Fig. 7. The corresponding fitCMD parameters are $M_{\mathrm{cl}}=(2.7 \pm 0.5) \times 10^{3} \mathrm{M}_{\odot}$ (only $\approx 8$ per cent of this mass is present on the observed CMD.), age $=13.0_{-0.9}^{+0.3} \mathrm{Gyr}, Z=0.0002$,

\footnotetext{
${ }^{3}$ https://datalab.noao.edu/query.php?name=des_dr1.main
}
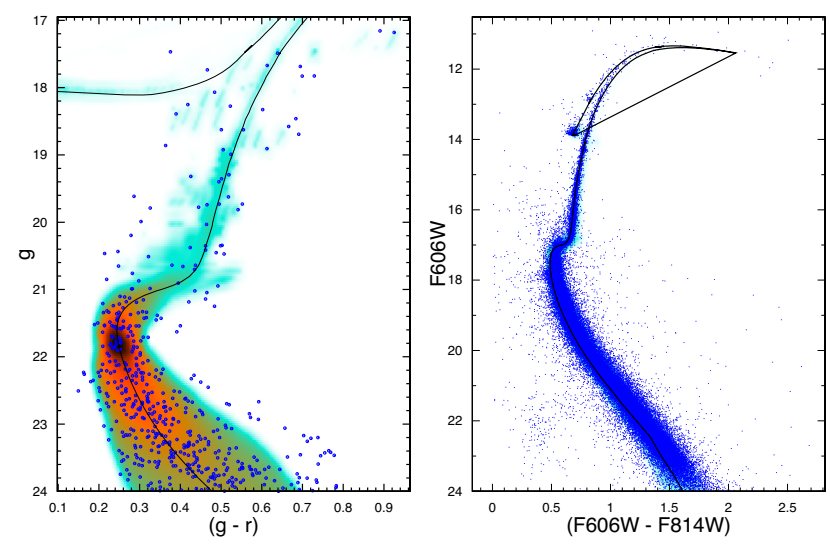

Figure 7. Left panels: same as Fig. 2 for the dwarf galaxy Ret II (left) and the GC 47 Tuc (right).
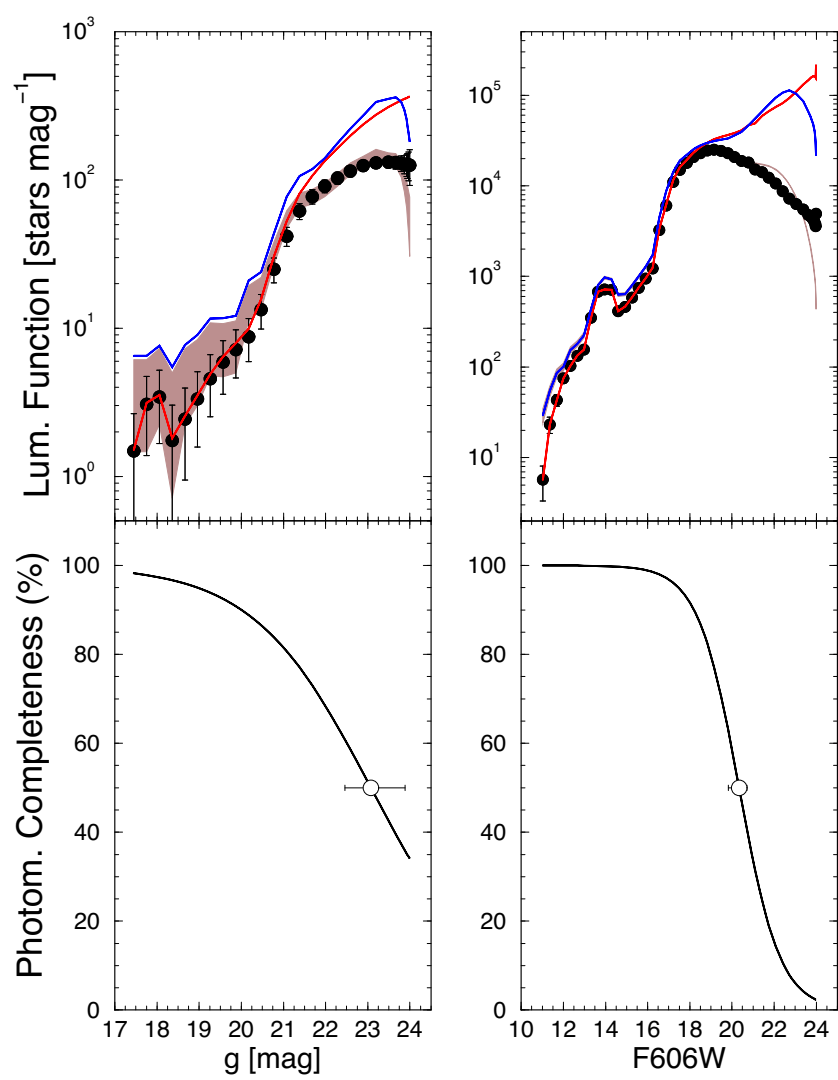

Figure 8. Top: same as Fig. 6 for Ret II (left) and 47 Tuc (right); bottom: same as Fig. 5.

$\mathrm{d}_{\odot}=30.6 \pm 1.4 \mathrm{kpc}$, the absolute magnitude in $g M_{g}=-3.2$, and the bolometric magnitude $M_{\mathrm{bol}}=-3.72$. $V$ magnitudes can be obtained from $g$ and $r$ by the transformation $V=g-0.487(g-$ $r)-0.025$ (Bechtol et al. 2015), resulting in $M_{V}=-3.5$. Within the quoted uncertainties, these parameters agree with the previous ones, except for the lower metallicity implied by Mutlu-Pakdil et al. (2018). Luminosity and photometric completeness functions are shown in Fig. 8. Consistently with ground-based observations of a distant object, Ret II photometry appears to be affected by completeness issues even at the bright end of the LF.

47 Tucanae (NGC 104) is the second brightest Milky Way GC after Omega Centauri (NGC 5139). Its tens of thousands stars dis- 
tribute across $\sim 50^{\prime}$ of the southern sky. 47 Tuc lies at $\mathrm{d}_{\odot} \approx 4.5 \mathrm{kpc}$ from the Sun, displays a small and dense central core $\left(R_{c} \approx 0^{\prime} .36\right)$ and has half-light and tidal radii of $R_{\mathrm{hl}} \approx 3.2^{\prime}$ and $r_{\mathrm{t}} \approx 43$ arcmin, respectively (Harris 2010). More recently, 47 Tuc was shown to host multiple stellar populations (Milone et al. 2012).

High-quality photometry for 47 Tuc is available as part of the HST WFC/ACS GC sample under program number GO 10775, with A. Sarajedini as PI. GO 10775 is a HST Treasury project in which 66 GCs were observed through the $F 606 \mathrm{~W}$ and $F 814 \mathrm{~W}$ filters with a field of view of $\approx 200 \times 200 \operatorname{arcsec}^{2}$ (Sarajedini et al. 2007). Working with this photometry, Wagner-Kaiser et al. (2017) found $A_{V}=$ $0.105 \pm 0.002,[\mathrm{Fe} / \mathrm{H}]=-0.72([\alpha / \mathrm{Fe}] \approx+0.4)$, which corresponds to $Z \approx 0.006$, and the rather old value age $=13.494_{-0.022}^{+0.006} \mathrm{Gyr}$. On the other hand, working with deep WFC3 IR photometry, Correnti et al. (2016) derived age $=11.6 \pm 0.7 \mathrm{Gyr}$. It is clear that there is no consensus on the exact age of 47 Tuc. Indeed, Brogaard et al. (2017) find the value $11.8 \mathrm{Gyr}$, with lower and upper limits $(3 \sigma)$ at 10.4 and $13.4 \mathrm{Gyr}$, a range that appears to realistically represent the actual age uncertainty of 47 Tuc. Concerning the stellar mass of 47 Tuc, $N$-body computations of Marks \& Kroupa (2010) provide $7 \times 10^{5} \mathrm{M}_{\odot}$, while the analysis of central velocity dispersions coupled to fits of dynamical models by Kimmig et al. (2015) implies $(7 \pm 1) \times 10^{5} \mathrm{M}_{\odot}$.

The fitCMD analysis of 47 Tuc is based on the Sarajedini et al. (2007) photometry; as an additional quality constraint, only stars with photometric uncertainty lower than 0.1 mag in F606W and F814W (reducing to $\approx 128000$ the number of available stars) are considered. The fitCMD solution for 47 Tuc is shown in Fig. 7, and the best-fitting parameters are $M_{\mathrm{cl}}=(3.4 \pm 0.4) \times 10^{5} \mathrm{M}_{\odot}$ $\left(\approx 24\right.$ per cent of this is on the observed CMD), age $=12.0_{-0.5}^{+0.3} \mathrm{Gyr}$, $Z=0.004, \mathrm{~d}_{\odot}=4.5 \pm 0.1 \mathrm{kpc}$, the absolute magnitude in $F 606 \mathrm{~W}$ $M_{F 606 W}=-8.34$, the bolometric magnitude $M_{\mathrm{bol}}=-8.63$, and $A_{V}$ $=0.11 \pm 0.01$. fitCMD age agrees quite well with the values of Brogaard et al. (2017) and Correnti et al. (2016). The fitCMD value for the mass corresponds to about half of the dynamical estimates (see above). However, it should be noted that the fitCMD value is based on the stars present in the area sampled by WFC/ACS, which has a radius corresponding to about half of $R_{\mathrm{hl}}$. Interestingly, photometric completeness is inconspicuous for $F 606 W \lesssim 16$, but falls off quite steeply for $F 606 \mathrm{~W} \gtrsim 18$ (Fig. 8).

\section{CONCLUDING REMARKS}

fitCMD is an approach that extracts a set of astrophysical parameters from CMDs of star clusters. The rationale is to transpose theoretical IMF properties to their observational counterpart, the CMD. This requires finding values of the total (or cluster) stellar mass, age, global metallicity, foreground reddening, distance modulus, as well as for parameters describing magnitude-dependent photometric completeness. These parameters - including photometric scatter - are used to build a synthetic CMD that is compared with that of a star cluster. Residual minimization between observed and synthetic CMDs - by means of the global optimization algorithm Simulated Annealing - then leads to the best-fitting parameters.

The efficiency of fitCMD, both in terms of computational time and ability to recover input parameters, has been tested with CMDs of artificial and observed star cluster - as well as of a dwarf galaxy and a GC, with excellent results. In principle, fitCMD can be used with any isochrone set that provides magnitudes for at least two different bands for stellar masses covering as wide as possible a range. In addition, the isochrone set should also provide a comprehensive coverage - and resolution - both in age and in metallicity.

\section{ACKNOWLEDGEMENTS}

Thanks to an anonymous referee for important comments and suggestions. This research has made use of the VizieR catalogue access tool, CDS, Strasbourg, France. This publication makes use of data products from the Two Micron All Sky Survey, which is a joint project of the University of Massachusetts and the Infrared Processing and Analysis Center/California Institute of Technology, funded by the National Aeronautics and Space Administration and the National Science Foundation. CB acknowledges support from the Brazilian Institution CNPq.

\section{REFERENCES}

Amin M. Y., Elsanhoury W. H., 2017, Serbian Astron. J., 194, 59

Bechtol K. et al., 2015, ApJ, 807, 50

Bessell M. S., 1990, PASP, 102, 1181

Bessell M. S., Brett J. M., 1988, PASP, 100, 1134

Bonatto C., Bica E., 2007, MNRAS, 377, 1301

Bonatto C., Bica E., 2011, MNRAS, 415, 2827

Bonatto C., Lima E. F., Bica E., 2012, A\&A, 540, A137

Bressan A., Marigo P., Girardi L., Salasnich B., Dal Cero C., Rubele S., Nanni A., 2012, MNRAS, 427, 127

Brogaard K., VandenBerg D. A., Bedin L. R., Milone A. P., Thygesen A., Grundahl F., 2017, MNRAS, 468, 645

Correnti M., Gennaro M., Kalirai J. S., Brown T. M., Calamida A., 2016, ApJ, 823, 18

Cummings J. D., Kalirai J. S., Tremblay P.-E., Ramirez-Ruiz E., 2016, ApJ, 818,84

Da Rio N., Gouliermis D. A., Gennaro M., 2010, ApJ, 723, 166

Goffe W. L., Ferrier G. D., Rogers J., 1994, J. Econometrics, 60, 65

Goodwin S. P., 2009, Ap\&SS, 324, 259

Goodwin S. P., Bastian N., 2006, MNRAS, 373, 752

Harris W. E., 2010, preprint (arXiv:1012.3224)

Heiter U., Soubiran C., Netopil M., Paunzen E., 2014, A\&A, 561, A93

Hess R., 1924, Probleme der Astronomie. Festschrift fur Hugo v. Seeliger. Springer, Berlin, p. 265

Hills S., von Hippel T., Courteau S., Geller A. M., 2015, AJ, 149, 94

Hurley J. R., Pols O. R., Aarseth S. J., Tout C. A., 2005, MNRAS, 363, 293

Kimmig B., Seth A., Ivans I. I., Strader J., Caldwell N., Anderton T., Gregersen D., 2015, AJ, 149, 53

Kroupa P., 2001, MNRAS, 322, 231

Lada C. J., Lada E. A., 2003, ARA\&A, 41, 57

Lamers H. J. G. L. M., Gieles M., 2006, A\&A, 455, L17

Lamers H. J. G. L. M., Baumgardt H., Gieles M., 2010, MNRAS, 409, 305

Marigo P. et al., 2017, ApJ, 835, 77

Marks M., Kroupa P., 2010, MNRAS, 406, 2000

Martinez-Medina L. A., Gieles M., Pichardo B., Peimbert A., 2018, MNRAS, 474, 32

Milone A. P. et al., 2012, ApJ, 744, 58

Moitinho A., Carraro G., Baume G., Vázquez R. A., 2006, A\&A, 445, 493

Montgomery K. A., Janes K. A., Phelps R. L., 1994, AJ, 108, 585

Mutlu-Pakdil B. et al., 2018, ApJ, 863, 25

Naylor T., Jeffries R. D., 2006, MNRAS, 373, 1251

Piatti A. E., Clariá J. J., Ahumada A. V., 2006, MNRAS, 367, 599

Salpeter E. E., 1955, ApJ, 121, 161

Sarajedini A. et al., 2007, AJ, 133, 1658

Sarajedini A., von Hippel T., Kozhurina-Platais V., Demarque P., 1999, AJ, 118,2894

Skrutskie M. F. et al., 2006, AJ, 131, 1163

Stead J. J., Hoare M. G., 2011, MNRAS, 418, 2219

Taylor B. J., 2007, AJ, 133, 370

Wagner-Kaiser R. et al., 2017, MNRAS, 468, 1038

Yadav R. K. S. et al., 2008, A\&A, 484, 609

This paper has been typeset from a $\mathrm{T}_{\mathrm{E}} \mathrm{X} / \mathrm{LAT} \mathrm{E}$ file prepared by the author. 